\title{
Mitophagy in carcinogenesis, drug resistance and anticancer therapeutics
}

\author{
Yanjie Guan ${ }^{1 \dagger}$, Yifei Wang ${ }^{1 \dagger}$, Bo Li ${ }^{1}$, Kai Shen ${ }^{1}$, Quanfu $\mathrm{Li}^{1,2}$, Yingyin $\mathrm{Ni}^{1,2^{*}}$ and Lei Huang ${ }^{1,2^{*}}$ (D)
}

\begin{abstract}
The mitochondrion is an organelle that plays a vital role in energy production, cytoplasmic protein degradation and cell death. Mitophagy is an autophagic procedure that specifically clears damaged mitochondria and maintains its homeostasis. Emerging evidence indicates that mitophagy is involved in many physiological processes, including cellular homeostasis, cellular differentiation and nerve protection. In this review, we describe the regulatory mechanisms of mitophagy in mammals and yeasts and highlight the recent advances relevant to its function in carcinogenesis and drug resistance. Finally, a section has been dedicated to describing the role of mitophagy in anticancer therapeutics, which is a new frontier that offers a precise and promising strategy.
\end{abstract}

Keywords: Mitophagy, Mechanism, Carcinogenesis, Drug resistance, Anticancer therapeutics

\section{Background}

The stability of mitochondria is essential for cellular homeostasis and diverse cellular functions [1-6]. Apart from the well-known role of intracellular energy factories through oxidative phosphorylation (OXPHOS) [1, 2], mitochondria are the organelles responsible for the production of reactive oxygen species (ROS), cytoplasmic protein degradation [3], maintenance of calcium homeostasis [4], heme biosynthesis [5], apoptotic activation [6] and innate immunity through mitochondrial antiviralsignaling protein (MAVS) [7].

Autophagy is an important process in cells for degrading proteins and organelles in a lysosome-dependent manner. Mitophagy refers to the process of degrading mitochondria through selective autophagy [8-10]. The concept of mitophagy was first proposed by the Lemasters group in 2005 [8]. Under the conditions of ROS

\footnotetext{
*Correspondence: niyingyin@126.com; leihuang@shsmu.edu.cn

†Yanjie Guan and Yifei Wang contributed equally to this work

1 Department of Histoembryology, Genetics and Developmental Biology,

Key Laboratory of Cell Differentiation and Apoptosis of Chinese Ministry of Education, Shanghai Key Laboratory of Reproductive Medicine,

Shanghai Jiao Tong University School of Medicine, 280 South Chongqing Road, Shanghai 200025, People's Republic of China

Full list of author information is available at the end of the article
}

stimulation, nutrition deficiency, and cell senescence, mitochondria undergo membrane potential depolarization $[8,11]$. Cellular proteins and depolarized mitochondria are sequestered in autophagosomes. Subsequently, autophagosomes fuse with lysosomes to degrade these contents and maintain the stability of the intracellular environment and mitochondrial fitness [8]. However, under severe conditions, in which mitophagy cannot handle a large number of damaged or dysfunctional mitochondria, cell death pathways are activated, and mitophagy is suppressed [12]. In addition to eliminating damaged mitochondria, researchers have identified numerous physiological and pathophysiological functions of mitophagy. Mitophagy contributes to cell development and erythrocyte differentiation. In mammals, the differentiation of erythrocytes relies on the removal of mitochondria by mitophagy [13]. When knocking out the mitophagy-related gene BCL2/adenovirus E1B $19 \mathrm{kDa}$ protein-interacting protein 3-like (BNIP3L) in mice, mitochondria accumulation occurs in erythrocytes, which results in anemia [14]. In adipocytes, mitophagy eliminates excessive mitochondria to promote a beige-to-white adipocyte transition [15]. Since mitophagy maintains mitochondrial homeostasis, it is not surprising that the dysregulation of mitophagy has a

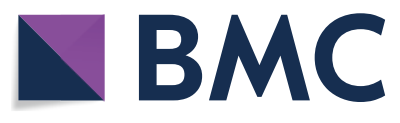

c) The Author(s) 2021. This article is licensed under a Creative Commons Attribution 4.0 International License, which permits use, sharing, adaptation, distribution and reproduction in any medium or format, as long as you give appropriate credit to the original author(s) and the source, provide a link to the Creative Commons licence, and indicate if changes were made. The images or other third party material in this article are included in the article's Creative Commons licence, unless indicated otherwise in a credit line to the material. If material is not included in the article's Creative Commons licence and your intended use is not permitted by statutory regulation or exceeds the permitted use, you will need to obtain permission directly from the copyright holder. To view a copy of this licence, visit http://creativeco mmons.org/licenses/by/4.0/. The Creative Commons Public Domain Dedication waiver (http://creativecommons.org/publicdomain/ zero/1.0/) applies to the data made available in this article, unless otherwise stated in a credit line to the data. 
role in human diseases. In the brain tissue of Parkinson's disease patients, there are excessive dysfunctional mitochondria, and the mutation of PTEN-induced putative kinase 1 (PINK1) was identified in this tissue [16]. Then PINK1 is proven to be a mitophagy-related gene [17]. Additionally, a similar phenomenon is found in Alzheimer's disease patients, indicating that mitophagy plays a protective role in neurodegenerative diseases [18]. The occurrence and development of cancers is a complicated pathophysiological process, and the effect of mitophagy on cancers will be discussed later.

In this review, we present a brief introduction to the main mechanisms of mitophagy regulation. We also elucidated the roles of mitophagy in carcinogenesis, drug resistance and anticancer therapeutics.

\section{Common mechanisms of mitophagy} Mitochondrial membrane receptors-mediated mitophagy Mitochondrial membrane receptors mainly include BCL2/adenovirus E1B $19 \mathrm{kDa}$ protein-interacting protein 3 (BNIP3), BNIP3L, FUN14 domain-containing protein 1 (FUNDC1), activating molecule in Beclin 1-regulated autophagy (AMBRA1), FK506-binding protein 8 (FKBP8), ATPase family AAA domain-containing protein 3B (ATAD3B), and some kinds of lipids (cardiolipin (CL) and C18-ceramide). These mitochondrial receptors depend on microtubule-associated protein 1 light chain 3 (LC3)-interacting region (LIR) motifs interacting with LC3 for mitochondrial clearance. They are regulated at the transcriptional or post transcriptional level under hypoxia and starvation conditions by kinases, phosphatases, glucocorticoids and other regulation factors.

BNIP3 and its homologous BNIP3L belong to the BH3only protein family and induce cell death and mitophagy [19-22]. Upon stress conditions, BNIP3 and BNIP3L are integrated into the outer membrane of mitochondria in the form of a homodimer [23-25]. BNIP3 binds to LC3 by its LIR motif to induce mitophagy in various mammalian cells. Phosphorylation at Ser17 and Ser24 near the LIR motif is important for BNIP3-LC3 interactions [26]. The kinase or phosphatase responsible for the phosphorylation at Ser17 and Ser24 is not yet clear. BNIP3L shares a more than $50 \%$ amino acid sequence similarity with BNIP3 [21]. BNIP3L is a mitochondrial receptor mediating mitochondrial elimination during erythrocyte maturation [14, 22, 27]. Under hypoxic conditions or mitochondrial stress, BNIP3L interacts with ATG8 family proteins (GABARAP1/LC3A) through its LIR motif. The interaction between BNIP3L and GABARAP1/LC3A leads to depolarized mitochondrial clearance upon reticulocyte maturation [22, 28, 29]. In BNIP3L-deficient cells, autophagosomal formation is still functional, but mitochondria are unable to fuse with autophagosomes [13,
$14,22,29]$ Mitophagy induced by BNIP3 and BNIP3L is regulated by hypoxia-inducible factor 1 -alpha (HIF-1 $\alpha$ ) [30-32]. The upregulation of HIF-1 $\alpha$ under hypoxic conditions can enhance the expression of BNIP3 by activating the transcription factor forkhead box O3 (FOXO3) [31]. Glucocorticoids repress PGC1 $\alpha$ expression in a glucocorticoid receptor (GR)-dependent manner and mitigate BNIP3L-dependent mitophagy [33].

FUNDC1 plays a distinct role in eukaryotic cells from BNIP3 and BNIP3L. A possible explanation for this might be that FUNDC1 is a mitophagy assistant under hypoxic conditions. FUNDC1 interacts with LC3 through the LIR motif at the cytosol-exposed $\mathrm{N}$-terminus for selective mitophagy to couple with the core autophagic machinery [34]. The phosphorylation of FUNDC1 at Tyr18 in the LIR motif by Src kinase inhibits mitophagy from occurring [34]. FUNDC1 can be ubiquitinated by membrane-associated ring- $\mathrm{CH}$-type finger 5 (MARCH5) and degraded to avoid unnecessary mitochondrial clearance [35]. In addition, kinases such as casein kinase 2 (CK2) and Unc51-like kinase 1 (ULK1) and phosphatases such as phosphoglycerate mutase 5 (PGAM5) regulate the phosphorylation state of FUNDC1 and functionally cooperate to regulate mitophagy [34-37]. Collectively, the phosphorylation state of FUNDC1 dictates its affinity to LC3 and subsequently influences the activation of mitophagy. The PGC-1 $\alpha$-NRF1 pathway is a crucial regulator in mitochondrial biogenesis. PGC-1 $\alpha$ and NRF1 also increase the expression of FUNDC1 to enhance mitophagy to promote mitochondrial turnover and maintain functional mitochondria [38]. Such a mechanism serves to maintain the balance between the quality and quantity of mitochondria.

Ambral can induce the depolarization of mitochondria which leads to functional mitophagy via a Parkinindependent pathway. Ambra1 binds to the E3 ubiquitin ligase HUWE1 to induce the ubiquitylation of mitofusin 2 (MFN2), a mitochondrial membrane protein, with an overall effect on mitophagy induction. After mitophagy induction, Ambra1 binds to LC3 to complete the autophagosome formation [39]. Ambra1 acts as an alternative mediator in PINK1/Parkin-mutant Parkinson's disease patients [39]. Ambra1 can also be recruited by Parkin during mitochondrial depolarization and activates class III PI3K to form autophagosomes around mitochondria [40].

$\mathrm{CL}$, a kind of lipid located at the inner mitochondrial membrane, is involved in mitochondrial metabolism. Its externalization enables its interaction with LC3 to induce subsequent mitophagy to protect cells from apoptotic cell death. This pathway could be induced by rotenone, staurosporine, and 6-hydroxydopamine [41]. Additionally, Cers1 generates C18-ceramide, a bioactive sphingolipid, 
and mediates its localization on the membrane of the mitochondria. After mitochondrial fission by dynaminrelated protein 1 (DRP1), ceramides interact with LC3BII to induce mitophagy [42].

FKBP8 is anchored in the outer membrane of mitochondria and acts as a mitophagy receptor. It recruits LC3A by its LIR motif to damaged mitochondria to mediate Parkin-independent mitophagy [43]. ATAD3B, a mitochondrial receptor, interacts with LC3 to induce parkin-independent mitophagy under stress conditions. ATAD3B-induced mitophagy promotes the clearance of damaged mitochondrial DNA (mtDNA) [44] (Fig. 1A).

The phenomenon of mitophagy was first discovered in yeasts in 2005 [8]. Similar to BNIP3/BNIP3L/FUNDC1 in mammals, autophagy-related protein 32 (Atg32) is a mitochondrial receptor protein in the outer membrane of mitochondria with a classic tetrapeptide sequence $\mathrm{W} / \mathrm{Y} /$ $\mathrm{XXI} / \mathrm{L} / \mathrm{V}$ in yeasts. Under nitrogen starvation, Atg32 is phosphorylated by CK2, especially at Ser114, and then its $\mathrm{N}$-terminus binds to autophagy-related protein 11 (Atg11) [45-48]. The Atg32-Atg11 interaction is crucial for mitochondrial recruitment to the preautophagosomal structure (PAS) [47, 48]. The Atg32 cytoplasmic domain forms a conjugation with autophagy-related protein 8 (Atg8) by its conserved motif to accelerate the process of autophagosomes engulfing the mitochondria. The autophagosomes and lysosomes eventually fuse to clear these contents [49, 50] (Fig. 1B).

\section{PINK1/Parkin-mediated mitophagy}

PINK1, an outer mitochondrial membrane protein, is sensitive to mitochondrial membrane depolarization. Parkin, named for its causal role in the pathogenesis of early-onset Parkinson's disease, is an E3 ubiquitin-protein ligase comprising five conserved domains, UBL, RING0, RING1, IBR, and RING2 [51, 52]. Under basal conditions, PINK1 is transported from the cytosol to the mitochondrial matrix through mitochondrial translocases, led by its N-terminal mitochondrial targeting sequence. When crossing the inner mitochondrial membrane, PINK1 is cleaved by matrix processing peptidase (MPP), mitochondrial protease presenilin-associated rhomboid-like protein (PARL), and ATPase family gene 3-like protein
2 (AFG3L2). Then the cleaved form of PINK1 is released back to the cytosol and degraded through the ubiquitinproteasome pathway [53-55]. When mitochondria are compromised and depolarized, PINK1 accumulates on their outer membrane and then recruits Parkin. PINK1 activates Parkin by phosphorylating Parkin on Thr175, Thr217, and Ser65 and thereby initiates mitophagy [56, 57]. Parkin ubiquitinates the mitochondrial proteins of the mitochondrial outer membrane (such as mitofusin 1 (Mfn1) and Mfn2) and promotes ubiquitin chain generation. PINK1 also phosphorylates ubiquitin at Ser65 to enhance the recruitment and activation of Parkin and thus constitutes a feed-forward mechanism to promote mitophagy [58-60] (Fig. 1C).

Several other ubiquitin-protein E3 ligases function in mitophagy in addition to Parkin. An E3 ligase called protein ariadne-1 homolog (ARIH1) is dependent on PINK1 to initiate mitophagy in the absence of Parkin [61]. Seven in absentia homolog 1 (SIAH-1), a conserved ubiquitin E3 ligase, promotes mitophagy by forming the PINK1synphilin-1-SIAH-1 complex in the absence of Parkin. Synphilin-1 is recruited to the damaged mitochondria by PINK1 to depolarize mitochondria and stabilize uncleaved PINK1. Synphilin-1 then recruits SIAH-1 to ubiquitinate mitochondrial proteins and promotes mitophagy [62].

BNIP3 and BNIP3L also recruit Parkin to the mitochondria. Parkin ubiquitinates multiple mitochondrial membrane proteins, including voltage-dependent anion channel 1 (VDAC1), Mfn1 and mitochondrial Rho GTPase (MIRO), which subsequently interact with p62. The p62 adaptor interacts with LC3 to induce mitophagy [63, 64]. Additionally, PINK1 can be stabilized by BNIP3 in its full-length form, which promotes its ability to recruit Parkin subsequently [65].

Although there are interconnections between these mitophagy pathways, significant differences can be noticed between PINK1/Parkin-mediated mitophagy and mitochondrial receptor-mediated mitophagy. The core mechanism of PINK1/Parkin-mediated mitophagy is the generation of ubiquitin chains recognized by autophagic receptors. However, mitochondrial receptors such as BNIP3 and FUNDC1 contain a conserved LIR motif

(See figure on next page.)

Fig. 1 Mechanism of mitophagy regulation in mammals $(\mathbf{A}, \mathbf{C})$ and yeasts $(\mathbf{B})$. A Mitophagy receptors mediate mitophagy under hypoxia or starvation in mammals. The proteins BNIP3, BNIP3L, FKBP8 and FUNDC1 on the outer member of mitochondria directly bind with LC3 through their LIR domains. Lipid CL externalizes from the inner mitochondrial membrane and interacts with LC3 to initiate mitophagy. B Atg proteins mediated mitophagy in yeasts. Under nitrogen starvation, the mitochondrial outer member receptor Atg32 is phosphorylated by CK 2 and interacts with Atg8 or Atg11 to promote mitophagy. C PINK1/Parkin-mediated mitophagy under mitochondrial depolarization in mammals. Mitochondrial stress blocks the internalization of PINK1. Accumulated PINK1 on the outer member of mitochondria recruits cytosolic Parkin to mitochondria and phosphorylates them. Then, the activated Parkin ubiquitinates the mitochondria outer member proteins such as Mfn 1/Mfn2. Adaptors containing LIR motifs (e.g., p62, OPTN, NBR1) recognize these polyubiquitinated proteins and connect to autophagosomes via LC3 
Hypoxia/Starvation
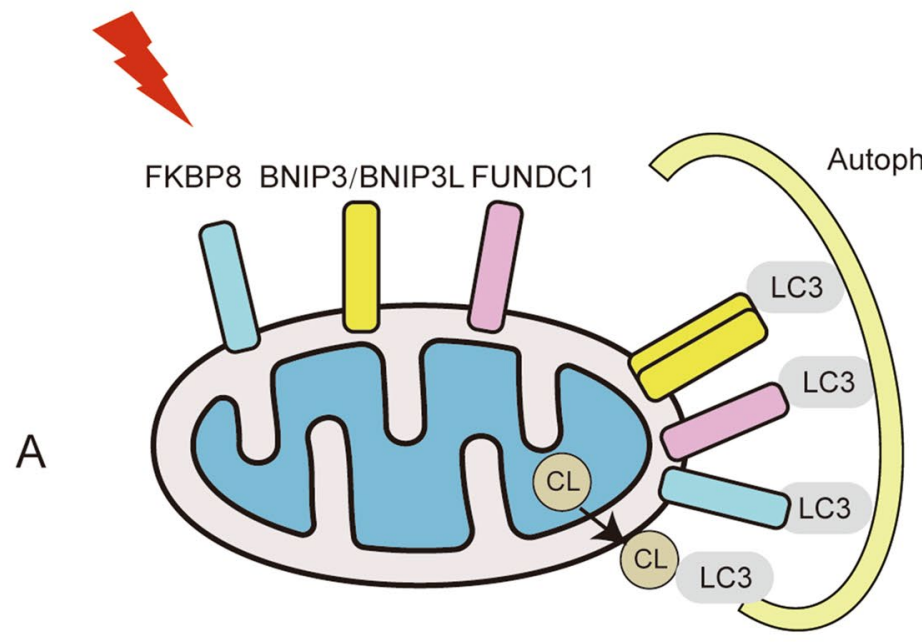

Nitrogen Starvation

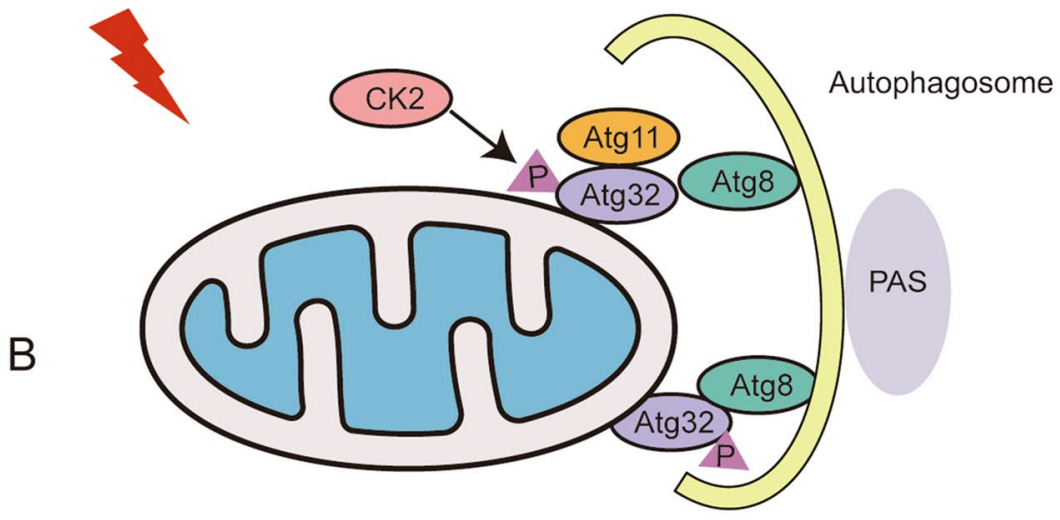

Mitochondria Depolarization

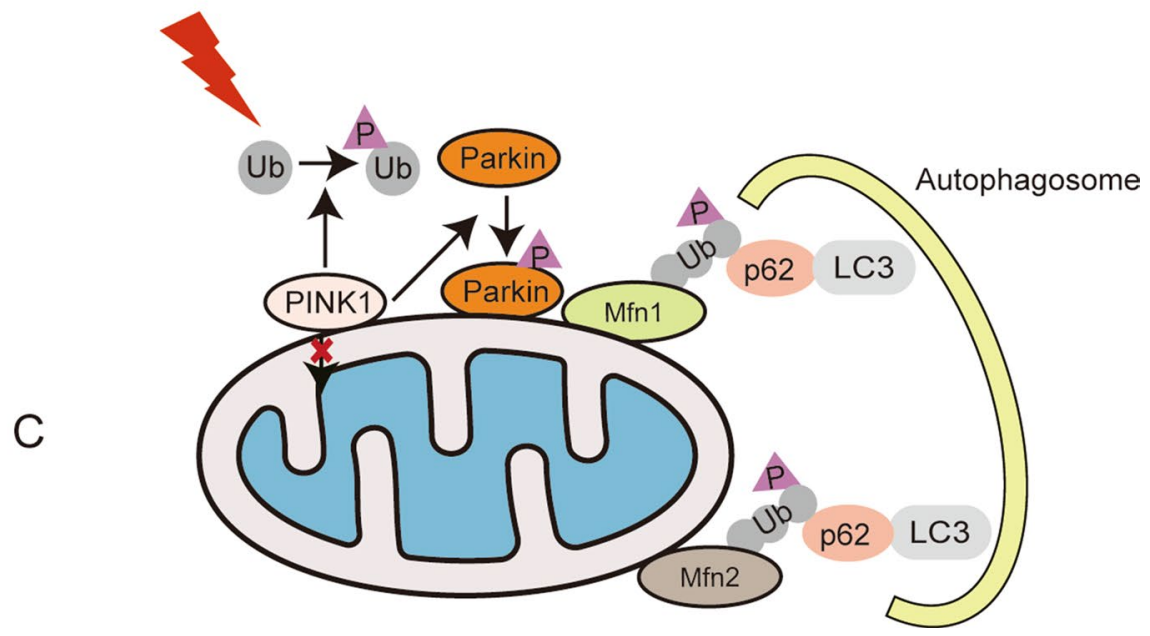

Fig. 1 (See legend on previous page.) 
and directly bind to LC3 on autophagosomes by the LIR motif.

\section{The role of mitophagy in carcinogenesis, drug resistance and cancer therapeutics Mitophagy and carcinogenesis}

Mitophagy plays a multifaceted role in carcinogenesis and cancer progression. Whether it behaves as a tumor promoter or suppressor largely depends on the statuses and subtypes of cancer cells [66, 67]. Mitophagy can remove damaged or dysfunctional mitochondria to maintain the balance between the quality and quantity of mitochondria. After mitophagy, functional mitochondria generate less ROS and limit the tumor-initiating capacity of ROS [68]. On the other hand, once the tumors are already in progress, mitophagy can function as a cytoprotective method to guide tumor progression against chemotherapy-induced apoptosis [69, 70]. Functional mitophagy inhibits the accumulation of damaged mitochondria and prevents carcinogenesis. A study in mouse hepatic cancer reported that thyroid hormone $T_{3}$ restrains carcinogenesis through activating the PINK1/ Parkin pathway [71]. The PINK/Parkin pathway can induce mitophagy in liver cells, remove dysfunctional mitochondria and reduce ROS generation. The deletion or mutation of genes such as PARK2 and BNIP3 can cause mitophagy inhibition, thereby promoting carcinogenesis and cancer progression [72-75]. Loss-of-function mutations in PARK2 gene that encodes Parkin, have been detected in human colorectal cancer. The overexpression of Parkin can inhibit the proliferation of colorectal cancer cells. In PARK2 heterozygous deletion mice, intestinal cancer development is accelerated [72]. FUNDC1-mediated mitophagy also inhibits inflammasome activation and protects against liver carcinogenesis. After the specific depletion of FUNDC1 in liver cells, dysfunctional mitochondria accumulate and therefore trigger inflammasome activation and carcinogenesis [76].

Cancer stem cells (CSCs), which form only a small proportion of the tumor cell population are closely related to the carcinogenesis, invasion, and the drug resistance of cancer. CSCs act as the bottleneck that restricts anticancer therapeutics. Mitophagy serves as a pro-survival pathway for CSCs. For example, in hepatic cancer, mitophagy can maintain the stemness and self-renewal ability of CSCs. Mitophagy can promote p53 degradation, which is combined with mitochondrial clearance. The inhibition of mitophagy leads to p53 transfer into the nucleus and blocks NANOG expression. Without this vital maintaining factor of CSC stemness, the hepatic CSC population is downregulated [77]. ISGylation of Parkin by ubiquitin-like protein ISG15 in pancreatic cancer stem cells (PaCSCs) promotes mitophagy to maintain
CSC self-renewal ability. Inhibition of ISG15 could result in reduced Parkin and impaired mitophagy, subsequently impairing PaCSC renewability and tumorigenesis capacity [70]. BNIP3L is highly expressed under hypoxic conditions. BNIP3L-mediated mitophagy promotes glioblastoma survival by clearing ROS and it may play a critical role in CSC maintenance [24].

\section{Mitophagy and drug resistance}

Certain chemotherapeutic drugs induce mitochondrial dysfunction, produce cytotoxic substances such as ROS, and influence normal metabolic activities [78-81]. Mitophagy is a cytoprotective process in the adaptation to chemotherapy drug treatment. Therefore, targeting mitochondria is regarded as a promising anticancer therapy.

Cisplatin is a widely used platinum-based compound that shows anticancer activity against various cancers. Cancers eventually develop resistance to cisplatin. Therefore, circumventing drug resistance is quite a challenge [61, 69, 82, 83]. Caveolin-1 (Cav-1)/Parkin-mediated mitophagy contributes to the resistance of the nonsmall cell lung cancer cell line A549 to cisplatin. The cav-1-knockdown A549 cells appear more sensitive to cisplatin because of the downregulated Rho-associated coiled-coil-containing protein kinase 1 (ROCK1) and subsequently suppresses Parkin-mediated mitophagy [82]. Apurinic endonuclease 1 (APE1) plays an important role in the cisplatin resistance of A549 cells, and APE1 is overexpressed in A549 cells and induces Parkin-mediated mitophagy. The knockdown of APE1 restores cisplatin sensitivity and promotes cell apoptosis [83]. In addition, another E3 ubiquitin ligase, ARIH1, is essential for initiating PINK1-dependent mitophagy in the absence of Parkin. ARIH-induced mitophagy acts as a defense mechanism against cisplatin-induced A549 cell death. In ARIH1 knockout cells, cisplatin at the same dose dominantly affects cell growth [61].

A new derivative of betulinic acid (BA), B5G1, has potent anticancer activity toward multidrug-resistant cancer cells by the induction of mitochondrial apoptosis. However, B5G1 can induce mitophagy through the upregulation of PINK1 and subsequent Parkin recruitment. The inhibition of mitophagy by mitochondrial division inhibitor 1 (mdivi-1) or bafilomycin sensitizes drug-resistant cancer cells to B5G1 [81].

Doxorubicin, a DNA damaging agent, greatly influences cell survival by inducing cell death and mitochondrial dysfunction. However, in colorectal cancer, damaged mitochondria are cleared by BNIP3L-mediated mitophagy to reduce oxidative stress and facilitate cell survival. The inhibition of mitophagy by BNIP3L 
knockout significantly improves its sensitivity to doxorubicin [69].

\section{Mitophagy as a target for anticancer therapeutics}

Mitophagy promotes cell survival by adapting to stress, but it may lead to cell death due to excessive mitochondrial clearance. Therefore, mitophagy inducers and inhibitors may be equally effective in anticancer treatment.

The inhibition of mitophagy plays a pivotal role in downregulating the drug resistance of cancer cells [78, 84, 85]. In cervical cancer, drug resistance to cisplatin suppresses the efficacy of chemotherapy. Melatonin (N-acetyl-5-methoxytryptamine) is an endogenous indoleamine and a famous antioxidant that reduces hypoxia-ischemia damage and improves sleep. It can also control tumor progression and inhibit mitophagy. Mechanistically, melatonin abates mitophagy by downregulating c-Jun N-terminal kinase (JNK) and subsequently Parkin, and it aggravates cervical cancer cell apoptosis [85]. In hepatic carcinoma, chemotherapeutic drugs such as cisplatin do not perform well in eliminating cancer cells due to inherent mitophagy and autophagy. Cisplatin activates dynamin-related protein 1 (DRP1) to enhance mitophagy. An inhibitor of DRP1-mediated mitophagy (mdivi-1) or a lysosome inhibitor (bafilomycin) increases the susceptibility of hepatic cancer cells to cisplatin rather than directly causing apoptosis [86]. Liensinine, an inhibitor of mitophagy, can markedly increase sensitivity to cisplatin in breast cancer. Liensinine inhibits mitophagy by suppressing the excessive accumulation of autophagosomes, autophagosome-lysosome fusion and the maturation of several important lysosomal hydrolases [87].
Enhanced mitophagy may lead to cell apoptosis due to insufficient functional mitochondria. Enhanced mitophagy can also provide a promising strategy for therapeutic intervention in other cancers. Ketoconazole, an oral antifungal agent, induces PINK1/Parkin-mediated mitophagy by downregulating the expression of COX2, which promotes the apoptosis of hepatic carcinoma cells [88]. In addition, sorafenib stimulates the apoptosis of liver cancer cells through PINK1/Parkin-mediated mitophagy. It stabilizes PINK1 on the outer membrane of mitochondria and recruits Parkin to dysfunctional mitochondria to initiate mitophagy [89]. Two mitochondria-targeted drugs, Mito-CP and Mito-Metformin, release ULK1 from mTOR-mediated inhibition, decrease mitochondrial membrane potential, and abrogate colorectal cancer cell proliferation through mitophagy [90] (Table 1).

\section{Conclusions}

With deep dissection of its role in carcinogenesis and drug resistance, mitophagy could become a breakthrough in cancer therapy. Whether these mitophagy inhibitors can be employed in clinical therapy for tumors? How to induce apoptosis of tumor cells rather than normal cells by regulation of mitophagy? In addition to endoplasmic reticulum stress, are there other organelles or physiological processes related to mitochondrial autophagy? Are there mitophagy inducers in specific tissues and cell types? How do normal cells sense the precisely regulated dynamic balance of mitochondria? A great deal of literature indicates that maintaining the balance between mitochondrial degradation and accumulation is essential for cellular homeostasis. Therefore, under certain conditions, the inhibition of mitophagy does more harm than good or has a slightly

Table 1 Mitophagy inhibitors and inducers in anticancer therapeutics

\begin{tabular}{|c|c|c|c|}
\hline No & Drug & $\begin{array}{l}\text { Mitophagy } \\
\text { inhibitor or } \\
\text { inducer }\end{array}$ & Mechanisms in anticancer therapeutics \\
\hline 1 & Melatonin & Inhibitor & Downregulates c-Jun N-terminal kinase (JNK) and subsequently Parkin, and aggravates cell apoptosis [85] \\
\hline 2 & Mdivi-1 & Inhibitor & $\begin{array}{l}\text { Increases the susceptibility of hepatic cancer cells to cisplatin by increasing mitochondrial membrane perme- } \\
\text { ability [86] }\end{array}$ \\
\hline 3 & Liensinine & Inhibitor & $\begin{array}{l}\text { Suppresses the excessive accumulation of autophagosomes, autophagosome-lysosome fusion and the matu- } \\
\text { ration of several important lysosomal hydrolases [87] }\end{array}$ \\
\hline 4 & Ketoconazole & Inducer & $\begin{array}{l}\text { Induces PINK1-Parkin mitophagy pathway by downregulating the expression of COX-2 and promotes the } \\
\text { apoptosis of hepatic carcinoma cells [88] }\end{array}$ \\
\hline 5 & Sorafenib & Inducer & $\begin{array}{l}\text { Stabilizes PINK1 on the outer membrane of mitochondria and recruits Parkin to dysfunctional mitochondria to } \\
\text { initiate mitophagy in liver cancer cells [89] }\end{array}$ \\
\hline 6 & $\begin{array}{l}\text { Mito-CP and } \\
\text { Mito-Met- } \\
\text { formin }\end{array}$ & Inducer & $\begin{array}{l}\text { Release ULK1 from mTOR-mediated inhibition, decrease mitochondrial membrane potential, and abrogate } \\
\text { colorectal cancer cell proliferation [90] }\end{array}$ \\
\hline
\end{tabular}


positive effect on the prognosis. As the energy factory of normal cells, the definitive role of mitochondria in cancer merits further systematic investigation. Identifying the precise role of mitophagy will provide an effective approach for mitophagy-based cancer therapy.

To develop new therapeutic strategies for rational anticancer therapy, further studies should pay more attention to the specific mechanisms of mitophagy in CSCs, such as the relationship between the amounts of mitochondria and the maintenance of CSCs. It is important to understand the interactions between oncogenic signaling pathways and mitophagy, the specific role of mitophagy in drug resistance, and the influences of mitophagy on different chemotherapeutic drugs. Mitophagy inducers or inhibitors should be delivered in a more targeted way. At the same time, with the development of novel drug therapies, it is important to be aware of the toxic effects derived from mitochondrial dysfunction and carefully consider them.

\begin{abstract}
Abbreviations
OXPHOS: Oxidative phosphorylation; ROS: Reactive oxygen species; MAVS: Mitochondrial antiviral-signaling protein; BNIP3L: BCL2 protein-interacting protein 3-like; PINK1: PTEN induced putative kinase 1; BNIP3: BCL2/adenovirus E1B 19 kDa protein-interacting protein 3; FUNDC1: FUN14 domain-containing protein 1; AMBRA1: Activating Molecule in Beclin 1-Regulated Autophagy; FKBP8: FK506-binding protein 8; ATAD3B: ATPase family AAA domain-containing protein 3B; CL: Cardiolipin; LC3: Microtubule-associated protein 1 light chain 3; LIR: LC3-interacting region; HIF-1a: Hypoxia-inducible factor 1-alpha; FOXO3: Forkhead box O3; GR: Glucocorticoid receptor; MARCH5: Membraneassociated ring-CH-type finger 5; CK2: Casein Kinase 2; ULK1: Unc51-like kinase 1; PGAM5: Phosphoglycerate mutase 5; Mfn2: Mitofusin 2; DRP1: Dynaminrelated protein 1; mtDNA: Mitochondrial DNA; Atg32: Autophagy-related protein 32; Atg 11: Autophagy-related protein 11; PAS: Pre-autophagosomal structure; Atg8: Autophagy-related protein 8; MPP: Matrix processing peptidase; PARL: Mitochondrial protease presenilin-associated rhomboid-like protein; AFG3L2: ATPase family gene 3-like protein 2; Mfn 1: Mitofusin 1; ARIH1: Protein ariadne-1 homolog; SIAH-1: Seven in absentia homolog 1;VDAC1: Voltage-dependent anion channel 1; MIRO: Mitochondrial Rho GTPase; CSCs: Cancer stem cells; PaCSCs: Pancreatic cancer stem cells; Cav-1: Caveolin-1; ROCK1: Rho-associated coiled-coil-containing protein kinase 1; APE1: Apurinic endonuclease 1; BA: Betulinic acid; Mdivi-1: Mitochondrial division inhibitor 1; JNK: C-Jun N-terminal kinase.
\end{abstract}

\section{Acknowledgements}

The authors wish to thank Liao XD, Deng HY and Liao $\mathrm{CH}$ for their assistance in construction and guidance. The authors wish to thank Zhu $\mathrm{Q}$ for his assistance in manuscript correction.

\section{Authors' contributions}

$\mathrm{LH}$, supervised the whole work and revised the manuscript, $\mathrm{YG}, \mathrm{YW}, \mathrm{BL}$ and KS made literature search and draft the manuscript of this review, QL and YN edited the manuscript. All authors read and approved the final manuscript.

\section{Funding}

The authors acknowledge financial support from National Natural Science Foundation of China (Grant Numbers 82073111, 81874197) and Science and Technology Commission of Shanghai Municipality (Grant Number 21S11901600) to L.H.

\section{Availability of data and materials}

Not applicable.

\section{Declarations}

Ethics approval and consent to participate Not applicable.

\section{Consent for publication}

All authors agree with the final version of the manuscript and give their consent for its publication.

\section{Competing interests}

The authors declare that they have no competing interests.

\section{Author details}

${ }^{1}$ Department of Histoembryology, Genetics and Developmental Biology, Key Laboratory of Cell Differentiation and Apoptosis of Chinese Ministry of Education, Shanghai Key Laboratory of Reproductive Medicine, Shanghai Jiao Tong University School of Medicine, 280 South Chongqing Road, Shanghai 200025, People's Republic of China. ${ }^{2}$ Innovative Research Team of High-Level Local Universities in Shanghai, Shanghai, People's Republic of China.

Received: 8 February 2021 Accepted: 29 June 2021

Published online: 05 July 2021

\section{References}

1. Lardy H, Ferguson S. Oxidative phosphorylation in mitochondria. Annu Rev Biochem. 1969:38:991-1034.

2. Wilson DF. Oxidative phosphorylation: unique regulatory mechanism and role in metabolic homeostasis. J Appl Physiol. 2017;122(3):611-9.

3. Heo JM, Livnat-Levanon N, Taylor EB, et al. A stress-responsive system for mitochondrial protein degradation. Mol Cell. 2010;40(3):465-80.

4. Robb-Gaspers LD, Burnett P, Rutter GA, et al. Integrating cytosolic calcium signals into mitochondrial metabolic responses. EMBO J. 1998;17(17):4987-5000.

5. Ponka P.Tissue-specific regulation of iron metabolism and heme synthesis: distinct control mechanisms in erythroid cells. Blood. 1997;89(1):1-25.

6. Matsuyama S, Llopis J, Deveraux QL, et al. Changes in intramitochondrial and cytosolic pH: early events that modulate caspase activation during apoptosis. Nat Cell Biol. 2000;2(6):318-25.

7. Seth R, Sun L, Ea C, et al. Identification and characterization of MAVS, a mitochondrial antiviral signaling protein that activates NF-kappaB and IRF 3. Cell. 2005;122(5):669-82.

8. Lemasters JJ. Selective mitochondrial autophagy, or mitophagy, as a targeted defense against oxidative stress, mitochondrial dysfunction, and aging. Rejuvenation Res. 2005;8(1):3-5.

9. Zhang $Y, X u X$, Hu M, et al. SPATA33 is an autophagy mediator for cargo selectivity in germline mitophagy. Cell Death Differ. 2021;28(3):1076-90.

10. Chang S, Lee A, Yu K, et al. Dihydroergotamine Tartrate Induces Lung Cancer Cell Death through Apoptosis and Mitophagy. Chemotherapy. 2016;61(6):304-12.

11. Livingston M, Wang J, Zhou J, et al. Clearance of damaged mitochondria via mitophagy is important to the protective effect of ischemic preconditioning in kidneys. Autophagy. 2019;15(12):2142-62.

12. Pagliarini V, Wirawan E, Romagnoli A, et al. Proteolysis of Ambra1 during apoptosis has a role in the inhibition of the autophagic pro-survival response. Cell Death Differ. 2012;19(9):1495-504.

13. Schweers RL, Zhang J, Randall MS, et al. NIX is required for programmed mitochondrial clearance during reticulocyte maturation. Proc Natl Acad Sci U S A. 2007;104(49):19500-5.

14. Sandoval H, Thiagarajan P, Dasgupta SK, et al. Essential role for Nix in autophagic maturation of erythroid cells. Nature. 2008;454(7201):232-5.

15. Lu X, Altshuler-Keylin S, Wang Q, et al. Mitophagy controls beige adipocyte maintenance through a Parkin-dependent and UCP1-independent mechanism. Sci Signal. 2018;11(527): eaap8526.

16. Nah J, Yuan J, Jung YK. Autophagy in neurodegenerative diseases: from mechanism to therapeutic approach. Mol Cells. 2015;38(5):381-9.

17. Valente EM, Abou-Sleiman PM, Caputo V, et al. Hereditary earlyonset Parkinson's disease caused by mutations in PINK1. Science. 2004;304(5674):1158-60. 
18. Manczak M, Kandimalla R, Yin X, et al. Hippocampal mutant APP and amyloid beta-induced cognitive decline, dendritic spine loss, defective autophagy, mitophagy and mitochondrial abnormalities in a mouse model of Alzheimer's disease. Hum Mol Genet. 2018;27(8):1332-42.

19. Chen G, Ray R, Dubik D, et al. The E1B 19K/Bcl-2-binding Protein Nip3 is a Dimeric Mitochondrial Protein that Activates Apoptosis. J Exp Med. 1997;186(12):1975-83.

20. Quinsay MN, Thomas RL, Lee Y, et al. Bnip3-mediated mitochondrial autophagy is independent of the mitochondrial permeability transition pore. Autophagy. 2010;6(7):855-62.

21. Matsushima M, Fujiwara T, Takahashi E, et al. Isolation, mapping, and functional analysis of a novel human CDNA (BNIP3L) encoding a protein homologous to human NIP3. Genes Chromosomes Cancer. 1998;21:230-5.

22. Zhang J, Ney P. NIX induces mitochondrial autophagy in reticulocytes. Autophagy. 2008:4(3):354-6.

23. Hanna RA, Quinsay MN, Orogo AM, et al. Microtubule-associated protein 1 light chain 3 (LC3) interacts with Bnip3 protein to selectively remove endoplasmic reticulum and mitochondria via autophagy. J Biol Chem. 2012:287(23):19094-104.

24. Jung J, Zhang Y, Celiku O, et al. Mitochondrial NIX Promotes Tumor Survival in the Hypoxic Niche of Glioblastoma. Cancer Res. 2019;79(20):5218-32.

25. Marinković M, Šprung M, Novak I. Dimerization of mitophagy receptor BNIP3L/NIX is essential for recruitment of autophagic machinery. Autophagy. 2021;17(5):1232-43.

26. Zhu Y, Massen S, Terenzio M. Modulation of Serines 17 and 24 in the LC3interacting Region of Bnip3 Determines Pro-survival Mitophagy versus Apoptosis. J Biol Chem. 2013;288(2):1099-113.

27. Chen M, Sandoval $H$, Wang J. Selective mitochondrial autophagy during erythroid maturation. Autophagy. 2008:4(7):926-8.

28. Schwarten M, Mohrlüder J, Ma P, et al. Nix directly binds to GABARAP: a possible crosstalk between apoptosis and autophagy. Autophagy. 2009;5(5):690-8

29. Novak I, Kirkin V, McEwan D, et al. Nix is a selective autophagy receptor for mitochondrial clearance. EMBO Rep. 2010;11(1):45-51.

30. Bellot G, Garcia-Medina R, Gounon P, et al. Hypoxia-induced autophagy is mediated through hypoxia-inducible factor induction of BNIP3 and BNIP3L via their BH3 domains. Mol Cell Biol. 2009;29(10):2570-81.

31. Aucello M, Dobrowolny G, Musarò A. Localized accumulation of oxidative stress causes muscle atrophy through activation of an autophagic pathway. Autophagy. 2009:5(4):527-9.

32. Sowter HM, Ratcliffe PJ, Watson P, et al. HIF-1-dependent regulation of hypoxic induction of the cell death factors BNIP3 and NIX in human tumors. Cancer Res. 2001;61(18):6669-73.

33. Gee EC, Hyun JL, Chang WC, et al. BNIP3L/NIX-mediated mitophagy protects against glucocorticoid-induced synapse defects. Nat Commun. 2021;12:487.

34. Liu L, Feng D, Chen G, et al. Mitochondrial outer-membrane protein FUNDC1 mediates hypoxia-induced mitophagy in mammalian cells. Nat Cell Biol. 2012;14:177-85.

35. Chen Z, Liu L, Cheng Q, et al. Mitochondrial E3 ligase MARCH5 regulates FUNDC1 to fine-tune hypoxic mitophagy. EMBO Rep. 2017;18(3):495-509.

36. Wu W, Tian W, Hu Z, et al. ULK1 translocates to mitochondria and phosphorylates FUNDC1 to regulate mitophagy. EMBO Rep. 2014;15(5):566-75.

37. Chen $G$, Han Z, Feng $D$, et al. A regulatory signaling loop comprising the PGAM5 phosphatase and CK2 controls receptor-mediated mitophagy. Mol Cell. 2014;54(3):362-77.

38. Liu L, Li YJ, Wang JN, et al. Mitophagy receptor FUNDC1 is regulated by PGC-1a/NRF1 to fine tune mitochondrial homeostasis. EMBO Rep. 2021;22(3):e50629.

39. Di Rita A, Peschiaroli A, Acunzo P, et al. HUWE1 E3 ligase promotes PINK1/ PARKIN-independent mitophagy by regulating AMBRA1 activation via IKKa. Nat Commun. 2018;9:3755.

40. Van $\mathrm{HC}$, Cornelissen $\mathrm{T}$, Hofkens $\mathrm{H}$, et al. Parkin interacts with Ambra1 to induce mitophagy. J Neurosci. 2011;31:10249-61.

41. Chu CT, Ji J, Dagda RK, et al. Cardiolipin externalization to the outer mitochondrial membrane acts as an elimination signal for mitophagy in neuronal cells. Nat Cell Biol. 2013;15(10):1197-205.
42. Sentelle RD, Senkal CE, Jiang W, et al. Ceramide targets autophagosomes to mitochondria and induces lethal mitophagy. Nat Chem Biol. 2012:8(10):831-8.

43. Bhujabal Z, Birgisdottir AB, Sjøttem E, et al. FKBP8 recruits LC3A to mediate Parkin-independent mitophagy. EMBO Rep. 2017;18(6):947-61.

44. Shu L, Hu C, Xu M, et al. ATAD3B is a mitophagy receptor mediating clearance of oxidative stress-induced damaged mitochondrial DNA. EMBO J. 2021;40(8):e106283.

45. Kanki T, Klionsky D, Okamoto K. Mitochondria autophagy in yeast. Antioxid Redox Signal. 2011;14(10):1989-2001.

46. Kondo-Okamoto N, Noda NN, Suzuki SW, et al. Autophagy-related protein 32 acts as autophagic degron and directly initiates mitophagy. J Biol Chem. 2012;287(13):10631-8.

47. Aoki Y, Kanki T, Hirota Y, et al. Phosphorylation of Serine 114 on Atg32 mediates mitophagy. Mol Biol Cell. 2011;22(17):3206-17.

48. Okamoto K, Kondo-Okamoto N, Ohsumi Y. Mitochondria-anchored receptor Atg32 mediates degradation of mitochondria via selective autophagy. Dev Cell. 2009;17(1):87-97.

49. Ichimura Y, Kumanomidou T, Sou Y, et al. Structural basis for sorting mechanism of p62 in selective autophagy. J Biol Chem. 2008;283(33):22847-57.

50. Kanki T, Furukawa K, Yamashita S. Mitophagy in yeast: Molecular mechanisms and physiological role. Biochim Biophys Acta. 2015;1853:2756-65.

51. Geisler S, Holmström KM, Skujat D, et al. PINK1/Parkin-mediated mitophagy is dependent on VDAC1 and p62/SQSTM1. Nat Cell Biol. 2010;12(2):119-31.

52. Kitada T, Asakawa S, Hattori N, et al. Mutations in the parkin gene cause autosomal recessive juvenile parkinsonism. Nature. 1998;392(6676):605-8.

53. Jin SM, Lazarou M, Wang C, et al. Mitochondrial membrane potential regulates PINK1 import and proteolytic destabilization by PARL. J Cell Biol. 2010;191(5):933-42.

54. Meissner $\mathrm{C}$, Lorenz $\mathrm{H}$, Weihofen $\mathrm{A}$, et al. The mitochondrial intramembrane protease PARL cleaves human Pink1 to regulate Pink1 trafficking. J Neurochem. 2011;117(5):856-67.

55. Yamano K, Youle R. PINK1 is degraded through the N-end rule pathway. Autophagy. 2013;9(11):1758-69.

56. Kim Y, Park J, Kim S, et al. PINK1 controls mitochondrial localization of Parkin through direct phosphorylation. Biochem Biophys Res Commun. 2008;377(3):975-80

57. Kondapalli C, Kazlauskaite A, Zhang N, et al. PINK1 is activated by mitochondrial membrane potential depolarization and stimulates Parkin E3 ligase activity by phosphorylating Serine 65. Open Biol. 2012;2(5):120080.

58. Kazlauskaite A, Kondapalli C, Gourlay R, et al. Parkin is activated by PINK1-dependent phosphorylation of ubiquitin at Ser65. Biochem J. 2014:460(1):127-39.

59. Ordureau A, Sarraf S, Duda D, et al. Quantitative proteomics reveal a feedforward mechanism for mitochondrial PARKIN translocation and ubiquitin chain synthesis. Mol Cell. 2014;56(3):360-75.

60. Koyano F, Okatsu K, Kosako H, et al. Ubiquitin is phosphorylated by PINK1 to activate parkin. Nature. 2014;510(7503):162-6.

61. Villa E, Proïcs E, Rubio-Patiño C, et al. Parkin-independent mitophagy controls chemotherapeutic response in cancer cells. Cell Rep. 2017:20(12):2846-59.

62. Szargel R, Shani V, Abd Elghani F, et al. The PINK1, synphilin-1 and SIAH-1 complex constitutes a novel mitophagy pathway. Hum Mol Genet. 2016;25(16):3476-90

63. Ding W, Ni H, Li M, et al. Nix is critical to two distinct phases of mitophagy, reactive oxygen species-mediated autophagy induction and Parkin-ubiquitin-p62-mediated mitochondrial priming. J Biol Chem. 2010;285(36):27879-90.

64. Lee $Y$, Lee $H$, Hanna R, et al. Mitochondrial autophagy by Bnip3 involves Drp1-mediated mitochondrial fission and recruitment of Parkin in cardiac myocytes. Am J Physiol Heart Circ Physiol. 2011;301(5):1924-31.

65. Zhang T, Xue L, Li L, et al. BNIP3 protein suppresses pink1 kinase proteolytic cleavage to promote mitophagy. J Biol Chem. 2016:291(41):21616-29.

66. Fei P, Wang W, Kim S, et al. Bnip3L is induced by p53 under hypoxia, and its knockdown promotes tumor growth. Cancer Cell. 2004;6(6):597-609.

67. Kulikov A, Luchkina E, Gogvadze V, et al. Mitophagy: Link to cancer development and therapy. Biochem Biophys Res Commun. 2017:482(3):432-9. 
68. Waris G, Ahsan H. Reactive oxygen species: role in the development of cancer and various chronic conditions. J Carcinog. 2006;5:14.

69. Yan C, Luo L, Guo C, et al. Doxorubicin-induced mitophagy contributes to drug resistance in cancer stem cells from HCT8 human colorectal cancer cells. Cancer Lett. 2017;388:34-42.

70. Im E, Yoo L, Hyun M, et al. Covalent ISG15 conjugation positively regulates the ubiquitin E3 ligase activity of parkin. Open Biol. 2016;6(8):160193.

71. Chi H, Chen S, Lin S, et al. Thyroid hormone protects hepatocytes from $\mathrm{HBx}$-induced carcinogenesis by enhancing mitochondrial turnover. Oncogene. 2017;36(37):5274-84.

72. Poulogiannis G, Mclntyre R, Dimitriadi M, et al. PARK2 deletions occur frequently in sporadic colorectal cancer and accelerate adenoma development in Apc mutant mice. Proc Natl Acad Sci U S A. 2010;107(34):15145-50.

73. He J, Pei L, Jiang H, et al. Chemoresistance of colorectal cancer to 5-fluorouracil is associated with silencing of the BNIP3 gene through aberrant methylation. J Cancer. 2017;8(7):1187-96.

74. Beroukhim R, Mermel C, Porter D, et al. The landscape of somatic copy-number alteration across human cancers. Nature. 2010;463(7283):899-905.

75. Cesari R, Martin E, Calin G, et al. Parkin, a gene implicated in autosomal recessive juvenile parkinsonism, is a candidate tumor suppressor gene on chromosome 6q25-q27. Proc Natl Acad Sci USA. 2003;100(10):5956-61.

76. Li W, LiY, Siraj S, et al. FUN14 domain-containing 1-mediated mitophagy suppresses hepatocarcinogenesis by inhibition of inflammasome activation in mice. Hepatology. 2019;69(2):604-21.

77. Liu K, Lee J, Kim J, et al. Mitophagy controls the activities of tumor suppressor p53 to regulate hepatic cancer stem cells. Mol Cell. 2017:68(2):281-92.

78. Carew J, Zhou Y, Albitar M, et al. Mitochondrial DNA mutations in primary leukemia cells after chemotherapy: clinical significance and therapeutic implications. Leukemia. 2003;17(8):1437-47.

79. Li N, Ragheb K, Lawler G, et al. Mitochondrial complex I inhibitor rotenone induces apoptosis through enhancing mitochondrial reactive oxygen species production. J Biol Chem. 2003;278(10):8516-25.

80. Lu J, Chew E, Holmgren A. Targeting thioredoxin reductase is a basis for cancer therapy by arsenic trioxide. Proc Natl Acad Sci USA. 2007;104(30):12288-93.
81. Yao N, Wang C, Hu N, et al. Inhibition of PINK1/Parkin-dependent mitophagy sensitizes multidrug-resistant cancer cells to B5G1, a new betulinic acid analog. Cell Death Dis. 2019;10(3):232.

82. Liu Y, Fu Y, Hu X, et al. Caveolin-1 knockdown increases the therapeutic sensitivity of lung cancer to cisplatin-induced apoptosis by repressing Parkin-related mitophagy and activating the ROCK1 pathway. J Cell Physiol. 2020;235(2):1197-208.

83. Li Z, Wang Y, Wu L, et al. Apurinic endonuclease 1 promotes the cisplatin resistance of lung cancer cells by inducing Parkin-mediated mitophagy. Oncol Rep. 2019;42(6):2245-54.

84. MacKeigan J, Murphy L, Blenis J. Sensitized RNAi screen of human kinases and phosphatases identifies new regulators of apoptosis and chemoresistance. Nat Cell Biol. 2005;7(6):591-600.

85. Chen L, Liu L, LiY, et al. Melatonin increases human cervical cancer HeLa cells apoptosis induced by cisplatin via inhibition of JNK/Parkin/ mitophagy axis. Vitro Cell Dev Biol Anim. 2018;54(1):1-10.

86. Ma M, Lin X, Liu H, et al. Suppression of DRP1-mediated mitophagy increases the apoptosis of hepatocellular carcinoma cells in the setting of chemotherapy. Oncol Rep. 2020;43(3):1010-8.

87. Zhou J, Li G, Zheng Y, et al. A novel autophagy/mitophagy inhibitor liensinine sensitizes breast cancer cells to chemotherapy through DNM1Lmediated mitochondrial fission. Autophagy. 2015;11(8):1259-79.

88. Chen Y, Chen H-N, Wang K, et al. Ketoconazole exacerbates mitophagy to induce apoptosis by downregulating cyclooxygenase- 2 in hepatocellular carcinoma. J Hepatol. 2019;70(1):66-77.

89. Zhang C, Liu Z, Bunker E, et al. Sorafenib targets the mitochondrial electron transport chain complexes and ATP synthase to activate the PINK1-Parkin pathway and modulate cellular drug response. J Biol Chem. 2017;292(36):15105-20.

90. Boyle K, Van Wickle J, Hill R, et al. Mitochondria-targeted drugs stimulate mitophagy and abrogate colon cancer cell proliferation. J Biol Chem. 2018;293(38):14891-904.

\section{Publisher's Note}

Springer Nature remains neutral with regard to jurisdictional claims in published maps and institutional affiliations.
Ready to submit your research? Choose BMC and benefit from:

- fast, convenient online submission

- thorough peer review by experienced researchers in your field

- rapid publication on acceptance

- support for research data, including large and complex data types

- gold Open Access which fosters wider collaboration and increased citations

- maximum visibility for your research: over $100 \mathrm{M}$ website views per year

At $\mathrm{BMC}$, research is always in progress.

Learn more biomedcentral.com/submissions 